\title{
HIV-1-specific CD4+ T lymphocyte turnover and activation increase upon viral rebound
}

\author{
Thomas J. Scriba, ${ }^{1}$ Hua-Tang Zhang, ${ }^{1}$ Helen L. Brown, ${ }^{1}$ Annette Oxenius, ${ }^{2}$ Norbert Tamm, ${ }^{3}$ \\ Sarah Fidler, ${ }^{3}$ Julie Fox, ${ }^{3}$ Jonathan N. Weber, ${ }^{3}$ Paul Klenerman,, ${ }^{1}$ Cheryl L. Day, ${ }^{1}$ \\ Michaela Lucas, ${ }^{1}$ and Rodney E. Phillips ${ }^{1}$
}

\begin{abstract}
${ }^{1}$ Peter Medawar Building for Pathogen Research and Nuffield Department of Clinical Medicine, University of Oxford, Oxford, United Kingdom. 2Institute for Microbiology, Eidgenössische Technische Hochschule Zurich, Zurich, Switzerland. ${ }^{3}$ Department of Medicine, Imperial College, St. Mary's Hospital, London, United Kingdom.
\end{abstract}

\begin{abstract}
HIV-specific CD4 ${ }^{+} \mathrm{T}$ helper lymphocytes are preferred targets for infection. Although complete interruption of combination antiretroviral therapy (ART) can form part of therapeutic manipulations, there is grave concern that the resumption of viral replication might destroy, perhaps irreversibly, these $T$ helper populations. High viremia blocks the proliferation capacity of HIV-specific helper cells. However, cytokine production assays imply that some antigen-specific effector function is retained. Despite this careful work, it remains unclear whether the return of HIV-1 replication physically destroys HIV-1-specific T helper cells in the peripheral blood. Difficulties in producing stable peptide-MHC class II complexes and the very low frequencies of antigen-specific $\mathrm{CD}^{+} \mathrm{T}$ cells have delayed the application of this powerful technique. Here we employ HLA class II tetramers and validate a sensitive, quantitative cell-enrichment technique to detect HIV-1 T helper cells. We studied patients with early-stage HIV infection who were given a short, fixed course of ART as part of a clinical study. We did not find significant deletion of these cells from the peripheral circulation when ART was stopped and unfettered HIV replication returned. The turnover of these virus-specific cells increased and they adopted an effector phenotype when viremia returned.
\end{abstract}

\section{Introduction}

$\mathrm{CD}^{+} \mathrm{T}$ lymphocytes are critical for immunological control of many viral infections, including HIV-1. Early studies showed that $\mathrm{HIV}$-specific $\mathrm{CD}^{+}$responses, as measured by proliferation assays, are meager or absent in most chronically HIV-infected patients $(1,2)$. Since $\mathrm{CD}^{+} \mathrm{T}$ cells are the principal target of HIV, and HIVspecific $\mathrm{CD}^{+}$cells are preferentially infected (3), this $\mathrm{T}$ helper population may be particularly vulnerable to destruction. There is grave concern that high HIV replication, such as the rebound viremia seen when combination antiretroviral therapy (ART) is stopped, might destroy HIV-specific $\mathrm{CD}^{+} \mathrm{T}$ cell populations permanently $(4,5)$. Several reports have shown substantial HIVspecific proliferative responses in patients who receive effective ART (6-8) and in long-term nonprogressors $(2,9)$. Also, sensitive cytokine (IFN- $\gamma$ ) assays show that HIV-specific CD4 ${ }^{+}$cells persist in most chronically infected patients $(2,10-12)$. Although HIVspecific proliferative responses are associated with viral control $(1,13)$, there are reported discrepancies between the numbers of HIV-specific IFN- $\gamma$-producing cells and proliferative capacity (2, 11). Furthermore, loss of proliferative capacity was shown to be associated with failure of HIV-specific IL-2 production (14-16). It has been hypothesized that this loss in proliferative capacity and IL-2 production is due to $\mathrm{CD}^{+} \mathrm{T}$ cell dysfunction and not to the physical loss of these $T$ cells.

Memory $\mathrm{CD}^{+} \mathrm{T}$ cells have been subdivided into central memory $T$ cells $\left(T_{c m}\right)$ and effector memory $T$ cells $\left(T_{e m}\right)$ based on the

Nonstandard abbreviations used: ART, antiretroviral therapy; $\mathrm{T}_{\mathrm{cm}}$, central memory $T$ cells; $\mathrm{T}_{\mathrm{em}}$, effector memory $\mathrm{T}$ cells.

Conflict of interest: The authors have declared that no conflict of interest exists.

Citation for this article: J. Clin. Invest. 115:443-450 (2005).

doi:10.1172/JCI200523084. expression of CCR7 and CD45RA. CD $4^{+} \mathrm{T}_{\mathrm{cm}}$ have been shown to produce predominantly IL-2 and have a high proliferative capacity, whereas $T_{\text {em }}$ produce mainly IFN- $\gamma$ and have limited proliferative capacity (17). In agreement with this, a skewing of the HIV-specific $\mathrm{CD}^{+}$memory population from $\mathrm{T}_{\mathrm{cm}}$ (CD45RA$\left.\mathrm{CCR}^{+} \mathrm{CD} 6 \mathrm{~L}^{+}\right)$towards $\mathrm{T}_{\mathrm{em}}$ (CD45RA-CCR7-CD62L-) in HIVinfected viremic individuals has been demonstrated $(15,16,18$, 19). These dysfunctional CD $4^{+}$cells may compromise $\mathrm{CD} 8^{+} \mathrm{CTL}$ activity and so erode immunity to HIV.

The study of antigen-specific $\mathrm{CD}^{+} \mathrm{T}$ cell function and phenotype is limited by requirements for in vitro stimulation before assaying cytokines or detecting proliferation. Although these methods have advanced our understanding of antigen-specific $\mathrm{CD}^{+} \mathrm{T}$ cells, it remains unknown whether or not there exist antigen-specific $\mathrm{CD}^{+} \mathrm{T}$ cell populations that do not produce the cytokines required for detection. These methods may bias analyses towards a limited subset of cytokine-producing cells. Also, in vitro stimulation is dependent on the activation of cells which results in altered expression of surface markers. For instance, upon antigenic stimulation, $T_{\mathrm{em}}$ transiently express CCR7 (20) and expression of CD62L on T cells in mice is rapidly lost (21). T cells can be directly visualized without antigen stimulation by staining them with fluorescently tagged MHC molecules folded around antigenic peptides. Peptide-MHC class I tetramers have greatly aided the study of antigen-specific $\mathrm{CD}^{+}$ cells. Although the generation of MHC class II tetramers has been more difficult, more sensitive methods for detecting very low cell frequencies have identified virus-specific $\mathrm{CD}^{+}$cells directly ex vivo $(22,23)$.

In this study, we employed MHC class II tetrameric complexes to enumerate and characterize Gag p 24 -specific $\mathrm{CD}^{+} \mathrm{T}$ cell populations ex vivo in HIV-infected individuals bearing HLA DRB1*0101. 

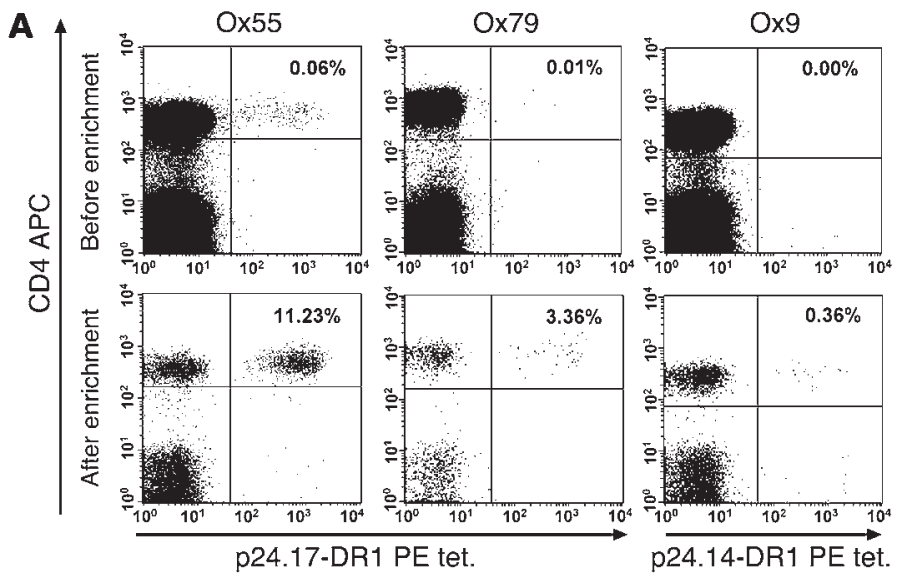

B
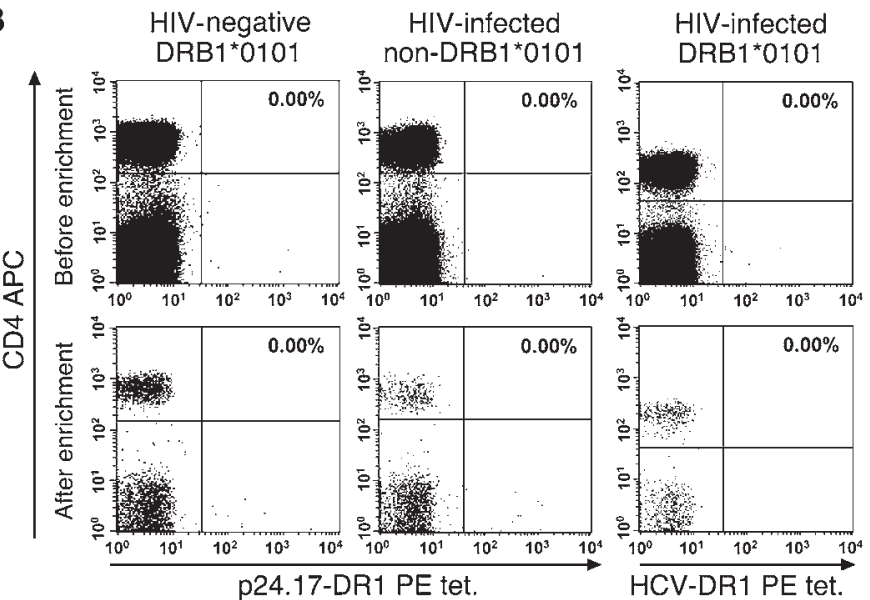

\section{Results}

Direct ex vivo detection of HIV Gag $p 24$-specific $C D 4^{+} T$ cells by HLA class II tetramer staining. We stained HIV-specific $\mathrm{CD}^{+} \mathrm{T}$ cells directly ex vivo with HLA class II tetramers. For confident detection of HLA class I-restricted cell populations in PBMCs, the lower limit is $0.02 \%$ of $\mathrm{CD}^{+}$cells $(24,25)$. This limit, which has been reported to also apply to HLA class II tetramer staining (26), is not sensitive enough for direct detection of most tetramer-positive $\mathrm{CD} 4^{+} \mathrm{T}$ cells in peripheral blood. To improve detection sensitivity, we employed a magnetic bead enrichment step $(22,23)$, which revealed tetramer-positive cells in 13 of the 14 patients tested (Figure 1A). To ensure that these tetramers were specific for $\mathrm{T}$ cells capable of recognizing HIV-1 p24 peptides bound to DRB ${ }^{*} 0101$, we stained fresh and cryopreserved PBMCs from healthy DRB1*0101 and

\section{Figure 2}

Validation of the linear recovery efficiency of the magnetic bead enrichment of tetramer-positive (tet $\left.{ }^{+}\right)$CD4 ${ }^{+} T$ cells. Uninfected PBMCs were spiked with a p24.17 epitope-specific CD4+ T cell clone, stained with the p24.17-DR1 tetramer, and subjected to magnetic bead enrichment. The observed frequencies (freq.) of tetramer ${ }^{+} \mathrm{CD} 4^{+}$cells versus the expected frequencies of tetramer ${ }^{+} \mathrm{CD} 4^{+}$cells are plotted. The FACS plots are shown in Supplemental Figure 1.

\section{Figure 1}

HLA class II tetramer staining of CD4+ $T$ cells. PBMCs were stained directly ex vivo and gated on CD14-, CD19-, and Via-Probe-negative cells. (A) Representative plots showing positive tetramer staining in HIV-infected individuals bearing DRB $1^{*} 0101$ stained with the HIV Gag p24.17-DR1 (panels Ox55 and Ox79 before and after enrichment with magnetic beads) and p24.14-DR1 (Ox9 before and after enrichment) PE-conjugated tetramers (PE tet). (B) Representative staining of control samples. Controls stained with the Gag p24-specific tetramers included HIV-negative individuals bearing DRB1*0101 (left, upper and lower panels) and HIV-infected non-DRB $1{ }^{*} 0101$ individuals (middle, upper and lower panels). HIV-infected individuals bearing DRB1*0101 were stained with a DRB1*0101 control tetramer complexed to an irrelevant HCV peptide (right, upper and lower panels). The percentage of cells falling into the upper-right quadrant is indicated for each panel.

HIV-infected non-DRB1*0101 individuals. No tetramer-positive cells were detected with either HIV-1-specific tetramer in 3 individuals who acted as controls (Figure 1B). The p24.17DR1 and P24.14-DR1 tetramers showed no staining in HIVnegative, $\mathrm{HCV}$-infected DRB $1{ }^{*} 0101$ patients (A. Ulsenheimer et al., unpublished observations). A DRB $1 * 0101$ tetramer complexed to an irrelevant $\mathrm{HCV}$ peptide was also employed as a control. No HCV tetramer staining was found in $3 \mathrm{HIV}$ infected, HCV-negative individuals bearing DRB1*0101 (Figure $1 \mathrm{~B}$ ), confirming that tetramer specificity was derived from both the peptide antigen and the HLA class II molecule.

Validation of quantitative linear recovery of tetramer-positive cell enrichment. To validate the reproducibility and linear recovery efficiency of the magnetic bead enrichment technique employed in this study, we spiked PBMCs from an HIV-negative donor with a 24.17 epitope-specific $\mathrm{CD}^{+} \mathrm{T}$ cell clone as described in Methods. When magnetic bead enrichment of tetramer-positive cells was used to quantify the number of tetramer-positive cells in each sample, a highly significant linear recovery was obtained $\left(r^{2}=0.9887, P<0.0001\right.$; Figure 2 and Supplemental Figure 1; supplemental material available online with this article; doi:10.1172/JCI200523084DS1). Further quantitative validation was achieved by tetramer staining and enrichment of multiple replicates from individual patients in parallel (data not shown). This analysis confirmed that tetramer-positive CD4 ${ }^{+}$ output frequencies were reproducible (SEM was consistently less than $11 \%$ of the mean response). Lucas et al. (23), who detected influenza virus hemagglutinin-specific CD $4^{+} \mathrm{T}$ cells directly ex vivo, had similar reproducibility.

In this study, we detected p24.17-DR1 and p24.14-DR1 tetramer-positive cells by direct ex vivo analysis at frequencies

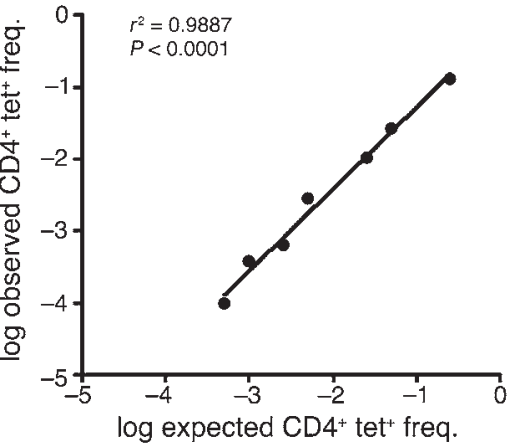


A

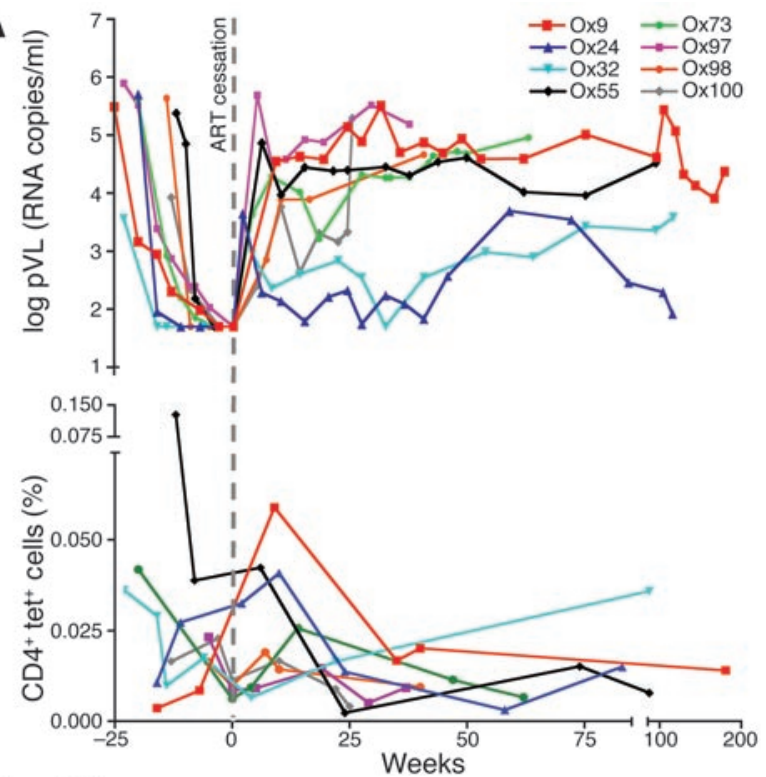

B

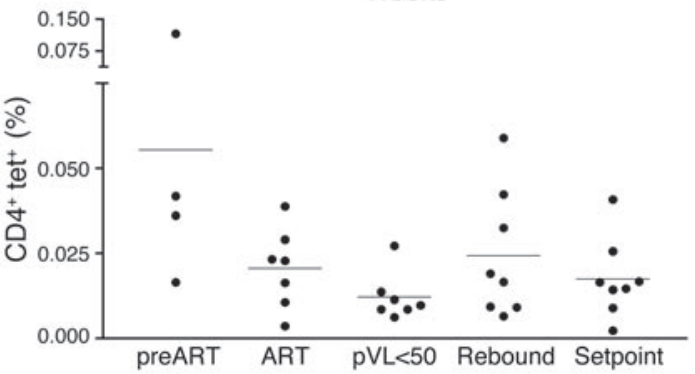

up to $0.14 \%$ and $0.008 \%$ of $\mathrm{CD}^{+} \mathrm{T}$ cells, respectively. To determine whether HIV-specific CD4 ${ }^{+} \mathrm{T}$ cells in PBMC samples from 6 patients with detectable p24.17-DR1 tetramer-positive cells were functional, we assayed for IFN- $\gamma$ and IL-2 production using a magnetic bead enrichment-based cytokine-secretion technique. Upon p24.17 Gag peptide stimulation, IFN- $\gamma$ production by $\mathrm{CD}^{+} \mathrm{T}$ cells was detected in all 6 patients tested, while IL- 2 was detected in 3 patients (see Supplemental Figure 2). The number of cytokine-producing $\mathrm{CD}^{+}$cells was found to be lower than that of tetramer-positive cells in all 6 patients.

Tetramer-positive $C D 4^{+} T$ cells survive when ART is stopped and viremia returns. HIV-specific $\mathrm{CD}^{+} \mathrm{T}$ cells lose their ability to proliferate and to produce IL-2 rapidly after primary HIV infection in most untreated viremic individuals $(1,2,11,14-16)$. The capacity for IFN- $\gamma$ production persists, as judged by intracellular cytokine staining and ELISpot assay (1, 2, 10-12, 14-16). To determine whether cessation of ART and the resultant viral rebound destroys $\mathrm{CD}^{+} \mathrm{T}$ cells specific for the p24.17 epitope, we studied 8 DRB $1 * 0101$-positive patients who had a short fixed course of ART that was stopped in line with a study protocol (27). We counted tetramer-positive $\mathrm{CD}^{+} \mathrm{T}$ cells during the following stages: acute infection, the viral downslope while on ART, undetectable viral load while on ART, the viral rebound that followed therapy cessation, and when the plateau after the initial viral rebound (viral setpoint) had been reached (Figure 3). The longest period between treatment cessation and followup procedures was 188 weeks. All 8 patients had undetectable plasma viral loads during ART (Figure $3 \mathrm{~A}$ ). No significant difference in

\section{Figure 3}

Effect of ART cessation on the physical numbers of Gag p24-specific CD4+ $T$ cells. PBMCs from 8 patients were stained with an HLA class II tetramer before, during, and after ART. (A) Longitudinal sampling of plasma viral load ( $p$ VL; top plot) and frequencies of p24.17-DR1 tetramer ${ }^{+}$cells out of total CD4 ${ }^{+} \mathrm{T}$ cells (bottom plot). ART cessation is represented by the vertical dashed line, and the data are normalized for therapy cessation (defined as week 0 ). (B) The frequencies of p24.17-DR1 tetramer-positive CD4+ $T$ cells are shown for 5 different clinical states: before antiretroviral therapy (preART), during the viral downslope while on ART (ART), once an undetectable viral load had been maintained for at least 1 month $(\mathrm{pVL}<50)$, upon viral rebound (Rebound), and during viral setpoint (Setpoint).

tetramer-positive $\mathrm{CD}^{+} \mathrm{T}$ cell frequencies was found when each of 5 phases of the rebound and its aftermath were compared (Figure 3B). Viral rebound did not result in significant destruction of circulating HIV-specific CD $4^{+} \mathrm{T}$ cell clones. In addition, tetramer-positive $\mathrm{CD}^{+}{ }^{+}$cells persisted in all 8 patients for at least 6 months and up to 188 weeks in patient Ox9. No patient showed complete depletion of these HIV-specific T helper populations. There was no correlation between tetramer-positive $\mathrm{CD}^{+}$cell frequencies and total $\mathrm{CD}^{+}$count or plasma viral load when all values were included in the analysis or when data during ART viral suppression were excluded (data not shown). We also measured $\mathrm{CD}^{+} \mathrm{T}$ cell responses to HIV antigens by ex vivo IFN- $\gamma$ ELISpot analysis of CD8-depleted PBMCs during the same 5 clinical states. In a patient cohort not selected for expression of DRB $1 * 0101(n=9)$, IFN- $\gamma$ responses revealed a similar result to the tetramer analysis; no significant change in HIV-specific CD4 ${ }^{+}$ $\mathrm{T}$ cell numbers was seen upon ART cessation and viral rebound (data not shown). These results show that rebound HIV viremia does not eliminate HIV-specific $\mathrm{CD}^{+} \mathrm{T}$ cell populations from the peripheral blood. We then investigated whether the renewed antigen drive altered the state of these cells.

Tetramer-positive, HIV-1-specific $\mathrm{CD}^{+} \mathrm{T}$ cells are effector cells. Although tetramer-positive cells remained intact during viral rebound, the resumption of HIV-1 replication might alter the function or turnover of this cell population. To examine this, we determined the phenotype of tetramer-positive $\mathrm{CD}^{+} \mathrm{T}$ cells in 9 patients by costaining for cell surface markers of memory (CD45RA), lymph node homing (CCR7 and CD62L), costimulation (CD27 and CD28), activation (CD38), and differentiation (CD57) (Figure $4 \mathrm{~A})$. In viremic individuals, HIV p24.17-specific cells were found

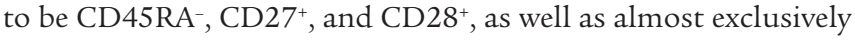
$\mathrm{CCR}^{-}$and $\mathrm{CD}^{-} 7^{-}$(Figure 4B). An intermediate expression pattern was seen for L-selectin (CD62L). CCR7 expression on tetramer-positive cells in viremic patients was also compared to the expression levels in the naive (CD45RA ${ }^{+}$) and memory (CD45RA-) CD4 ${ }^{+} \mathrm{T}$ cell subsets (Figure 4C). This analysis reveals significantly lower surface expression of CCR7 on HIV-specific tetramer-positive CD $4^{+}$ cells (median 12.5\%) compared to the memory $\mathrm{CD}^{+} \mathrm{T}$ cell population (median 51.8\%). Both HIV-specific tetramer-positive CD4 ${ }^{+}$ cells and total memory $\mathrm{CD} 4^{+}$cells showed significantly less CCR7 expression than naive $\mathrm{CD}^{+}{ }^{+} \mathrm{T}$ cells (median 95.1\%).

The median expression of CD38 was found to be approximately $47 \%$ on tetramer-positive cells, suggesting a high level of $\mathrm{T}$ cell activation. When the level of CD38 expression was compared between HIV-specific tetramer-positive cells and the total mem-

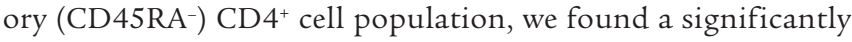



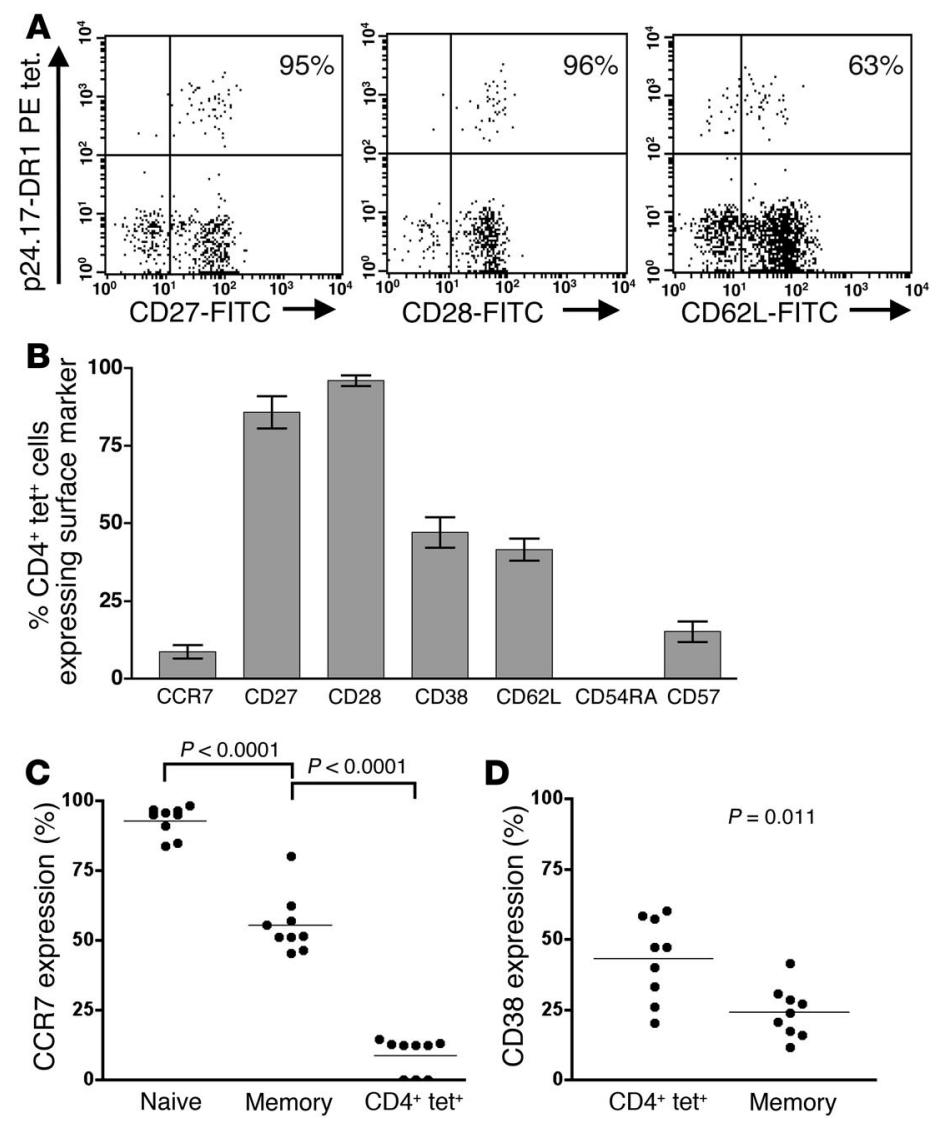

lower level of CD38 expression on the latter (median 23.7\%; $P=0.011$; Figure 4D). This significance was upheld when the CD38 mean fluorescence index between the same populations was compared (data not shown).

Elevated turnover of HIV-specific $\mathrm{CD}^{+} \mathrm{T}$ cells during viremia. HIVspecific $\mathrm{CD}^{+} \mathrm{T}$ cells are preferentially infected (3), and they are probably destroyed as a result of the infection. However, we did not find a significant decrease in the numbers of HIV-specific tetramer-positive $\mathrm{CD}^{+} \mathrm{T}$ cell clones after viral rebound. A possible explanation for the maintenance of virus-specific helper cells is an increased turnover of these cells to compensate for the loss incurred during high viremia. Indeed, elevated $\mathrm{CD}^{+}$and $\mathrm{CD}^{+}$ cell turnover, as measured by intracellular Ki67 staining, BrdU incorporation, and/or $\left[{ }^{2} \mathrm{H}\right]$ glucose in viremic HIV infections, has been reported (28-33). To test whether HIV-specific $\mathrm{CD}^{+} \mathrm{T}$ cells display elevated turnover rates, we measured intracellular Ki67 expression on tetramer-positive $\mathrm{CD}^{+} \mathrm{T}$ cells. In 10 viremic

\section{Figure 5}

Expression of the intracellular proliferation marker Ki67. (A) Representative costaining of CD4+ $\mathrm{T}$ cells with the p24.17-DR1 tetramer and $\mathrm{Ki} 67$ in 2 viremic patients. The percentage of tetramer+ cells expressing Ki67 is indicated in the upper-right quadrant. (B) Ki67 expression in $\mathrm{CD}^{+}{ }^{+} \mathrm{T}$ cell subsets in viremic infection. CD45RA ${ }^{+}$and CD45RA- CD4 ${ }^{+}$ cells were gated on to distinguish between the naive and memory subsets, respectively. The means are represented by horizontal lines and the statistical differences between them were calculated with a paired Student's $t$ test. (C) Correlation between plasma viral load and Ki67 expression in HIV-specific tetramer+ $\mathrm{CD}^{+}$cells.

\section{Figure 4}

Phenotypic analysis of HIV-specific memory CD4+ $\mathrm{T}$ cells using HLA class II tetramers. (A) Representative phenotypic staining with anti-CD27, anti-CD28, or anti-CD62L antibodies from subject Ox73. FACS plots are gated on CD4+ $\mathrm{T}$ lymphocytes, and the percentage of tetramer ${ }^{+} \mathrm{CD} 4^{+} \mathrm{T}$ cells expressing the respective phenotypic marker is indicated in the upper right quadrant of each plot. (B) Phenotypic profile of HIV-specific CD4+ $\mathrm{T}$ cells in 9 viremic individuals. Each bar represents the mean percentage of tetramer+ ${ }^{+} D 4^{+}$cells that express the indicated surface marker. Error bars represent SEM. (C) CCR7 expression on CD4+ $\mathrm{T}$ cell subsets in viremic HIV infection. CD45RA ${ }^{+}$and CD45RA- CD4+ cells were gated on to distinguish between the naive and memory subsets, respectively. (D) The HIV-1-specific, tetramer ${ }^{+}$ $\mathrm{CD}^{+} \mathrm{T}$ cell subset was significantly more activated than the memory $\mathrm{CD}^{+} \mathrm{T}$ cell population. CD38 expression was measured in 9 viremic patients. Cells that fell into the CD45RA-CD4+ or tetramer ${ }^{+} \mathrm{CD}_{4}{ }^{+}$gates were analyzed. The means are represented by horizontal lines and the statistical differences between them were calculated with a paired Student's $t$ test.

patients, we found significantly higher Ki67 expression on tetramer-positive $\mathrm{CD}^{+} \mathrm{T}$ cells than on memory (CD45RA-)

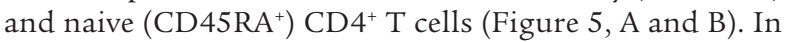
addition, a significant direct correlation was found between the frequency of $\mathrm{Ki}^{+} 7^{+}$tetramer-positive $\mathrm{CD} 4^{+}$cells and plasma viral load $\left(r^{2}=0.358, P=0.024\right.$; Figure 5C).

Correlation between plasma viral load and CCR7 expression on p24-specific tetramer-positive CD4 ${ }^{+} T$ cells. HIV-specific CD4 ${ }^{+}$ $\mathrm{T}$ cells have been shown to possess proliferative capacity in patients with very low viral replication and those who are ART suppressed (2, 6-9). During HIV viremia, antigen-specific proliferation $(1,2)$ and IL-2 production (14-16) are lost. To further determine the effects of HIV viremia, we quantified CCR7 on tetramer-positive cells. Tetramer-positive $\mathrm{CD} 4^{+} \mathrm{T}$ cells in patients receiving ART expressed significantly higher levels of CCR7 than those in viremic patients $(P=0.0021$; Figure 6 , A and B). Furthermore, a significant inverse correlation was found between the percentage of CCR7-expressing tetramer-positive cells and plasma viral load $\left(r^{2}=0.5033, P=0.0021\right.$; Figure 6C).
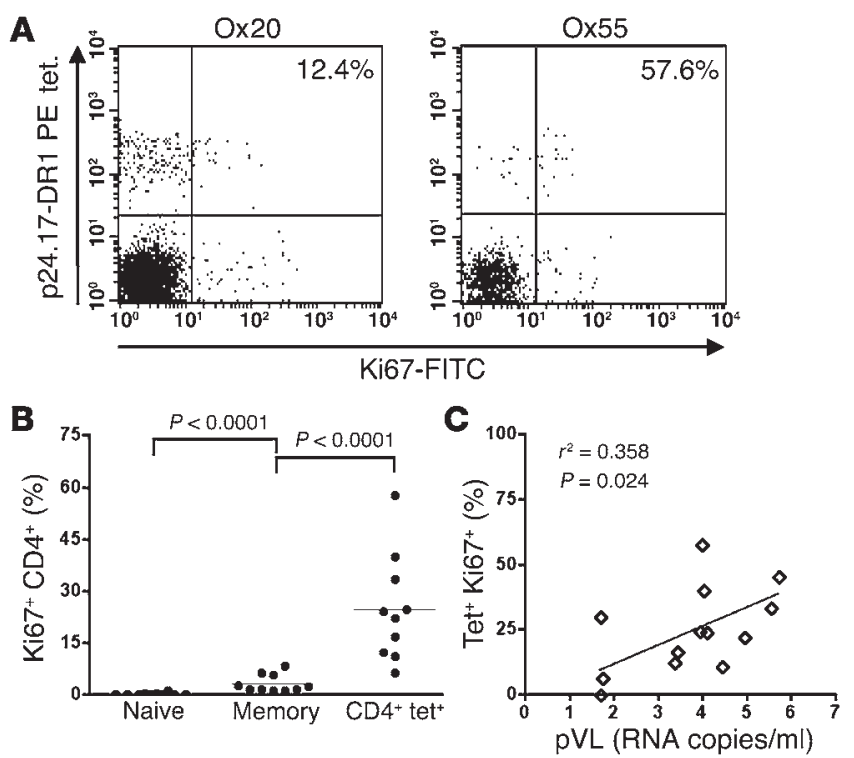

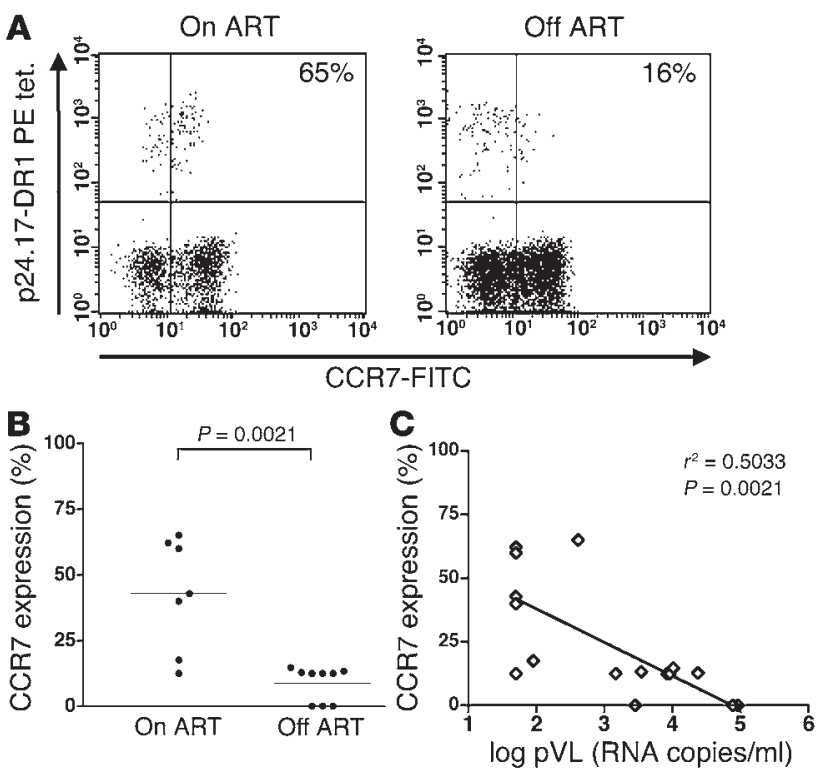

Tetramer-positive $\mathrm{CD}^{+}$cells in untreated chronically infected patients resemble those in patients treated acutely. To investigate whether these findings can be extended to chronically infected patients, we studied 2 ART naive DRB1*0101 patients (Figure 7). Longitudinal staining of PBMCs with p24.17-DR1 tetramer showed detectable frequencies of helper $T$ cell populations up to 63 and 85 weeks following the onset of seroconversion symptoms (Figure 7A). In these treatment-naive patients, cell phenotypes were very similar to those found following viral rebound (Figure 7).

\section{Discussion}

While HLA class I tetramers have revolutionized the study of antigen-specific $\mathrm{CD}^{+} \mathrm{T}$ cell frequencies and phenotypes, application of the same technology to the study of $\mathrm{CD}^{+} \mathrm{T}$ cells has been more difficult. Initial efforts to make stable HLA class II peptide-MHC complexes were frustrating and the low frequencies of antigen-specific CD $4^{+}$cells in peripheral blood were not readily detectable by direct ex vivo staining $(34,35)$. In this study we used a magnetic bead enrichment technique to enhance the sensitivity of HLA class II tetramer staining.

We consistently detected HIV Gag p24-specific CD4 ${ }^{+} \mathrm{T}$ cells in HIV-infected individuals at frequencies below the detection limit of direct flow cytometric analysis $(0.02 \%)(24,25)$. We demonstrated a linear recovery efficiency of the tetramer-

\section{Figure 7}

HIV-specific tetramer ${ }^{+} \mathrm{CD} 4^{+}$cells in untreated chronic patients resemble those in viremic patients who received short-course ART at acute infection. (A) Longitudinal detection of tetramer ${ }^{+}$ CD4+ cells up to 63 weeks and 85 weeks after the first experience of seroconversion symptoms in patients Ox22 and Ox79, respectively. The frequency of tetramer ${ }^{+} \mathrm{CD} 4^{+}$cells for each time point is indicated in the upper-right quadrant of each plot. (B) Phenotypic analysis of tetramer ${ }^{+} \mathrm{CD} 4^{+}$cells from patient Ox79. These analyses were performed at time points 52 weeks or more after the first experience of seroconversion symptoms. The percentage of tetramer+ ${ }^{+} D 4{ }^{+}$cells expressing the relevant marker is indicated in parentheses in each plot.

\section{Figure 6}

Effect of plasma viral load on CCR7 expression on HIV-specific CD4 ${ }^{+}$ T cells. (A) Representative FACS plots showing CCR7 staining on tetramer-positive CD4+ $T$ cells from patient Ox98 while on ART and off ART. Plots are gated on CD4+ lymphocytes. The percentages of tetramer+ ${ }^{+}$cells expressing CCR7 are indicated in the upper-right quadrant. (B) Comparison of percentage of CCR7 expression on tetramer+ CD4 ${ }^{+}$T cells in patients on ART $(n=7)$ and off ART $(n=9)$. The means are represented by horizontal lines and the statistical differences between them were calculated with the Mann-Whitney $U$ test. (C) Correlation between plasma viral load and percentage of CCR7 expression on tetramer-positive CD4 ${ }^{+} \mathrm{T}$ cells.

enrichment technique. Quantitative analysis using HLA-class II tetramer staining and magnetic bead enrichment to detect influenza HA-specific $\mathrm{CD}^{+}{ }^{+} \mathrm{T}$ cells was also highly reproducible (23).

Because HIV-specific CD $4^{+} \mathrm{T}$ cells are preferentially infected (3), there is concern that high levels of HIV replication, such as the rebound viremia seen when ART is stopped, might destroy HIVspecific helper $T$ cells $(4,5)$. In resource-poor settings short-course ART has been recommended by the World Health Organization for the prevention of mother-to-child transmission. Unscheduled cessation of ART is also likely to occur where medical supervision is limited or absent. Using HLA class II tetramer staining we directly measured HIV-specific T helper cell numbers in patients while on ART, during and after ART cessation. Our analyses showed no significant destruction of HIV-specific $\mathrm{CD}^{+}{ }^{+} \mathrm{T}$ cell clones when HIV viremia rebounded. This finding was confirmed by IFN- $\gamma$ ELISpot analysis of CD8-depleted PBMCs. These data correspond with previous studies that quantified HIV-specific IFN- $\gamma \mathrm{CD}^{+} \mathrm{T}$ cell numbers and demonstrated increased responses in patients during viremia compared to ART treatment $(14,15,36)$. Our analyses did not rule out the possibility that viral recrudescence could cause a redistribution of lymphocytes.
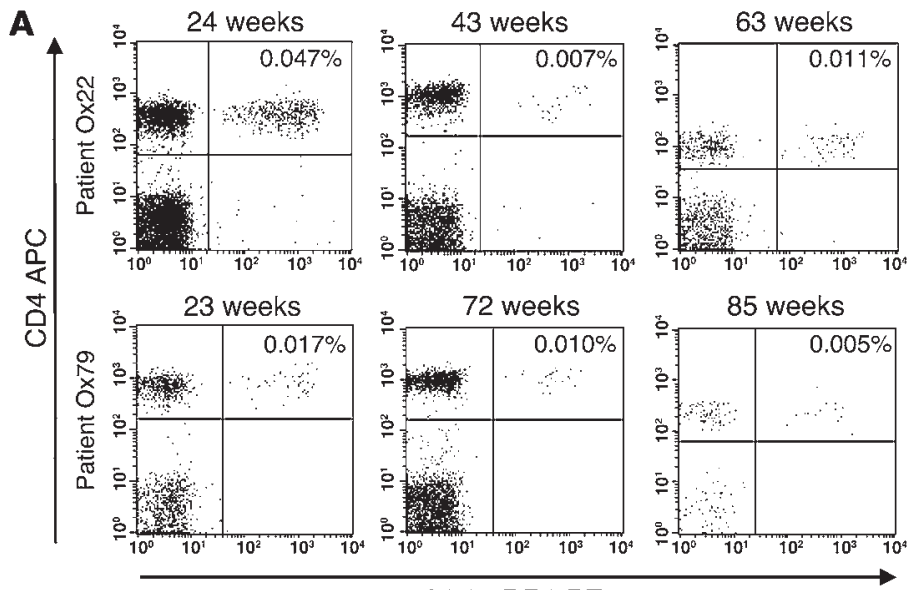

p24.17-DR1 PE tet.

\section{B}

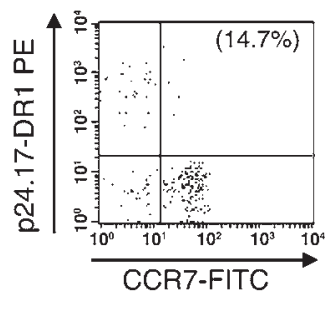

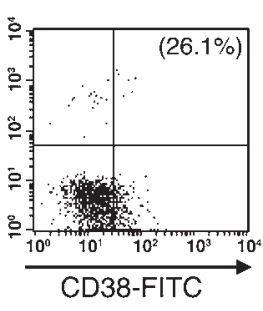

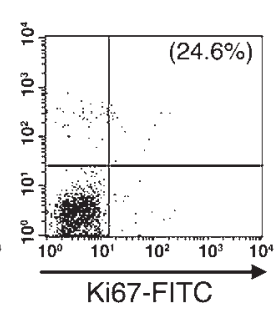




\section{Table 1}

Patient characteristics

\begin{tabular}{|c|c|c|c|}
\hline Patient & ART & $\begin{array}{l}\text { ART duration } \\
\text { (mo) }\end{array}$ & HLA DRB1 type \\
\hline $0 \times 9$ & Combivir, Nevirapine & 5 & ${ }^{\star} 0101,{ }^{\star} 0701$ \\
\hline $0 \times 19$ & Combivir, Nevirapine & 3 & *0101, *1101 \\
\hline $0 \times 20$ & Combivir, Nevirapine & 3 & *0101, *1501 \\
\hline $0 \times 22$ & untreated & NA & ${ }^{\star} 0101,{ }^{*} 1101$ \\
\hline $0 \times 24$ & Combivir, Nevirapine & 3 & ${ }^{\star} 0101,{ }^{*} 0102$ \\
\hline $0 \times 26$ & Combivir, Nevirapine & 5 & *0101, *0102 \\
\hline $0 \times 30$ & Combivir, Nevirapine & 5 & ${ }^{\star} 0101,{ }^{*} 0701$ \\
\hline $0 \times 32$ & Combivir, Nevirapine & 3 & *0101, *0701 \\
\hline $0 \times 55$ & Trizivir, Efavirenz & 2 & *0101, *0301 \\
\hline $0 \times 73$ & Trizivir, Efavirenz & 4 & ${ }^{\star} 0101,{ }^{\star} 0403$ \\
\hline $0 \times 79$ & untreated & NA & ${ }^{\star} 0101,{ }^{*} 0301$ \\
\hline $0 \times 97$ & Combivir, Kaletra & 4 & ${ }^{\star} 0101,{ }^{*} 0401$ \\
\hline $0 \times 98$ & Trizivir, Efavirenz & 3 & ${ }^{\star} 0101,{ }^{*} 0701$ \\
\hline $0 \times 100$ & Combivir, Kaletra & 3 & *0101, *0102 \\
\hline
\end{tabular}

HIV-specific proliferative responses significantly decrease in viremic patients $(2,11)$ along with virus-specific $\mathrm{CD}^{+}$responses as measured by IL-2 production (14-16). Our phenotypic analysis of HIV-specific tetramer-positive $\mathrm{CD}^{+}$cell clones reveals a dominant phenotype of CD45RA-CCR7- representing effector cells and an inverse correlation between CCR7 expression and plasma viral load. Low CCR7 expression was also demonstrated on MHC class II tetramer-positive $\mathrm{CD}^{+}{ }^{+}$cells during acute $\mathrm{HCV}$ infection (A. Ulsenheimer et al., manuscript submitted for publication).

These profiles contrast with those found on tetramer-positive $\mathrm{CD}^{+} \mathrm{T}$ cells specific for influenza or hepatitis $\mathrm{C}$ virus after infection has resolved; these $\mathrm{CD} 4^{+} \mathrm{T}$ helper cells display central memory phenotypes (CD45RA-CCR $\left.7^{+} \mathrm{CD} 62 \mathrm{~L}^{+}\right)(22,23)$. Our data suggest an "antigen dose"-dependent reduction in CCR7 expression on HIV-specific CD4 ${ }^{+} \mathrm{T}$ cells and are consistent with a recent study in which an inverse correlation was found between

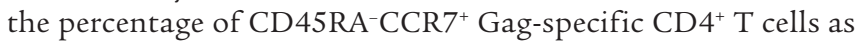
detected by intracellular cytokine staining (ICS) and plasma viral load (16). These data are consistent with analyses which relied on antigen-induced cytokine production in HIV-specific CD $4^{+}$ $\mathrm{T}$ cells. In viremic patients only a small percentage of these cells were able to produce IL-2, while most produced IFN- $\gamma$ (14-16, 18), a cytokine profile typical of effector cells (17). Harari et al. (19) and Younes et al. (15) also found low CCR7 expression on HIV-specific $\mathrm{CD}^{+} \mathrm{T}$ cells using an ICS assay. IFN- $\gamma^{+} \mathrm{HIV}$-specific $\mathrm{CD} 4^{+}$cells are increased in patients with viremia compared to treated HIV infection $(14-16,18)$. This may be the result of an increased capacity of effector cells to produce IFN- $\gamma$ rather than an increase in overall cell numbers. These and our results therefore fit into the current paradigm of central and effector phenotypes and their respective functional capacities as described by Sallusto et al. (17). Assays based on IFN- $\gamma$ detection may not be as useful for monitoring important immune responses as those measuring antigen-specific $\mathrm{CD}^{+}$and $\mathrm{CD}^{+}$lymphoproliferation and, in the case of $\mathrm{CD}^{+}$cells, IL-2 production.

Elevated turnover of total or memory $\mathrm{CD} 4^{+} \mathrm{T}$ cell populations in viremic HIV infection as measured by intracellular Ki67 staining, BrdU incorporation, and/or $\left[{ }^{2} \mathrm{H}\right]$ glucose has been described (29-33). In addition, a direct correlation between plasma viral load and Ki67 expression in the total $\mathrm{CD}^{+} \mathrm{T}$ cell pool was found (32). To determine whether high viral replication induces elevated turnover of HIV-specific CD $4^{+} \mathrm{T}$ cell populations, we measured intracellular Ki67 expression in the tetramer-positive cell population. HIV Gag p24-specific CD4 ${ }^{+}$cell clones had higher Ki67 expression than the memory $\mathrm{CD}^{+}$population. Ki67 expression in virus-specific tetramer-positive $\mathrm{CD}^{+}{ }^{+}$cells also correlated directly with plasma viral load. This increased cell turnover may explain the maintenance of HIV-specific $\mathrm{CD}^{+}$cell numbers in the face of high viral replication such as rebound viremia.

This viral load-dependent effect on $\mathrm{T}$ cell differentiation and turnover is likely to be the result of antigenic stimulation when HIV replication returns. The high level of activation (Figure 4, $\mathrm{B}$ and D; CD38 expression) seen in the HIV-specific CD4 ${ }^{+} \mathrm{T}$ cell compartment supports this. These data are compatible with the finding that $\mathrm{CD}^{+}$and $\mathrm{CD}^{+}$activation as measured by CD38 expression are correlated directly with viral load and predictive of changes in total CD4 counts (37).

Although viral recrudescence does not significantly alter the quantity of HIV-1-specific helper T cell populations in peripheral blood, function may be compromised and may contribute to the impaired immunity found in untreated HIV infection. When HIV rebounds, HIV-specific CD4 ${ }^{+} \mathrm{T}$ cells adopt the same phenotype as those found in untreated chronic HIV infection. Control of viral replication is dependent on functional HIV-specific $\mathrm{CD}^{+}{ }^{+} \mathrm{T}$ cells which can proliferate and produce IL-2 $(1,2)$, qualities attributed to $\mathrm{CD} 4 \mathrm{RA}{ }^{-} \mathrm{CCR} 7^{+}$cells. $\mathrm{HIV}$-specific $\mathrm{CD} 4^{+} \mathrm{CCR} 7^{+}$cells may be preserved by short-course ART for acute HIV infection. Postponement of the HIV-specific CD4+ T lymphocyte "functional switch" by a course of ART may translate as better long-term viral control. Our study supports evaluation of early treatment. A recent study showed that HIV-specific $\mathrm{CD}^{+}$proliferative responses returned in progressive infection when proliferative HIV-specific $\mathrm{CD} 4^{+}$helper cell responses were restored by therapeutic vaccination in patients receiving ART (38). Only controlled trials can determine if these interventions produce durable improvements in HIV-1 control.

In conclusion, we detected $\mathrm{HIV}$-specific $\mathrm{CD}^{+} \mathrm{T}$ cells directly ex vivo with HLA class II tetramers in patients on ART and after these drugs were stopped. This avoided a reliance on detection systems based on cytokine production, which require antigen stimulation and therefore might alter the phenotype and/or proliferation marker expression of these cells artefactually $(20,21,39)$. HIV-1specific $\mathrm{CD}^{+} \mathrm{T}$ cell populations survive viral rebound physically intact, show increased activation and turnover, and acquire an effector phenotype wholly consistent with the return of antigen drive characteristic of untreated HIV-1 infection.

\section{Methods}

Study population. We recruited 105 patients with acute HIV infection at St. Mary's Hospital, London. All but 16 patients received a short pulse of ART, after which therapy was stopped completely. These therapeutic interventions during acute infection have been demonstrated to preserve virus-specific $\mathrm{CD}^{+}$and $\mathrm{CD}^{+}$immune responses $(13,27,38,40,41)$ and have been advocated for clinical use. Resumption of therapy constituted a study endpoint for that patient (27). From this open study we investigated 14 patients who expressed HLA-DRB1*0101 (Table 1). Two of these patients opted to remain untreated, while 12 received ART for a period of 2 to 6 months. Informed consent was obtained from all participants and the study was given appropriate ethical approval by the Local Research Ethics Committee at St. Mary's Hospital Trust (99/IA/161E). 
HLA typing and viral load determination. High-resolution HLA class I and II genotypes were determined for each patient by PCR using sequencespecific primers (42). Plasma viral load was quantified using the Amplicor RT-PCR kit (Roche Diagnostic Systems). The lower limit of detection was 50 HIV RNA copies/ml.

Cell lines and clones. Fresh PBMCs were depleted of $\mathrm{CD}^{+}$cells using antiCD8 conjugated Dynabeads (Dynal UK Ltd.) and stimulated with $10 \mu \mathrm{g} / \mathrm{ml}$ peptide in medium containing $10 \%$ human $\mathrm{AB}+$ serum, $0.4 \mu \mathrm{M}$ Indinavir (Merck) and $0.5 \mu \mathrm{M}$ Zidovudine (GlaxoSmithKline). The plates were incubated at $37^{\circ} \mathrm{C}$ in $5 \% \mathrm{CO}_{2}$ and after 3 days the medium was supplemented with $100 \mathrm{U} / \mathrm{ml} \mathrm{IL-2} \mathrm{(Proleukin)} \mathrm{and} \mathrm{5 \%} \mathrm{human} \mathrm{T} \mathrm{cell} \mathrm{culture} \mathrm{supplement}$ (TSTIM; BD). Medium exchanges were done as necessary and after 12-14 days, cell lines were restimulated with $10 \mu \mathrm{g} / \mathrm{ml}$ peptide, IL-2, TSTIM, and irradiated autologous PBMCs. For generation of $\mathrm{CD} 4{ }^{+} \mathrm{T}$ cell clones, cell lines were stimulated with peptide and cells producing IFN- $\gamma$ and IL-2, which were selected using a cytokine detection and enrichment method (Miltenyi Biotech). IFN- $\gamma^{+}-$and IL- $2^{+}$-specific $\mathrm{CD}^{+}$cells were plated at limiting dilution in medium containing $10 \%$ human $\mathrm{AB}+$ serum, $2 \times 10^{5}$ irradiated mixed lymphocytes, $100 \mathrm{U} / \mathrm{ml} \mathrm{IL-2,5 \%} \mathrm{TSTIM,} \mathrm{and} 1 \mu \mathrm{g} / \mathrm{ml}$ phytohemagglutinin (PHA, Sigma-Aldrich).

IFN- $\gamma$ ELISpot assays for detection of $\mathrm{CD}^{+}$responses. Direct ex vivo IFN- $\gamma$ ELISpot analysis was performed as previously described (27). Briefly, fresh PBMCs were depleted of $\mathrm{CD} 8{ }^{+} \mathrm{T}$ cells using anti-CD8 conjugated Dynabeads. The efficiency of the CD8 depletion was tested routinely by FACS staining and less than $0.5 \%$ of $\mathrm{CD}^{+} \mathrm{T}$ cells were consistently detected (data not shown). Cells were stimulated in duplicate wells with recombinant proteins (p24, gp120, and p66 at $10 \mu \mathrm{g} / \mathrm{ml}$; National Institute for Biological Standards and Controls [NIBSC]) and overlapping pooled peptides (20-mers overlapping by 10; Tat and Nef at $5 \mu \mathrm{g} / \mathrm{mL}$ ). The HIV-unrelated recall antigens streptokinase and streptodornase (200 U/ml; Lederle), CMV lysate (10 $\mu \mathrm{g} / \mathrm{ml}$; Virusys), and PHA were used as positive controls. Nonrecombinant baculovirus and medium were used as negative controls. Spot quantification was automated and standardized with an ELISpot plate reader (Autoimmun Diagnostika).

Tetramer and antibody staining. The MHC class II tetramers used in this study were purchased from Beckman Coulter. The $2 \mathrm{HIV}$-specific tetramers were HLA-DRB1*0101 alleles complexed to the peptides p24.17 (AA 294-313, FRDYVDRFYKTLRAEQASQD; ref. 14) and p24.14 (AA 264-283, KRWIILGLNKIVRMYSPTSI; ref. 1). A DRB1*0101 class II tetramer complexed to an $\mathrm{HCV}$ peptide was used as peptide control tetramer (kindly donated by A. Ulsenheimer, University of Munich, Munich, Germany). Fresh or cryopreserved PBMCs were stained for 2 hours at room temperature with PE-conjugated MHC class II tetramer. Antibodies and Via-Probe (all from $\mathrm{BD}$, CCR7-FITC from R\&D Systems) were added during the last 30 minutes of incubation. The cells were washed and labeled with anti-PE microbeads (Miltenyi Biotech) and 10\% of the cells were reserved for FACS analysis, while $90 \%$ were subjected to magnetic bead enrichment of PE-conjugated tetramer-positive cells using MACS columns (Miltenyi Biotech).
For intracellular staining, cells were permeabilized using BD FixPerm (BD) following magnetic bead staining, stained with Ki67-FITC (Novocastra) and then applied to the MACS columns. Negative CD25 staining on tetramer-positive cells confirmed that the 2-hour tetramer staining did not activate the $\mathrm{CD}^{+} \mathrm{T}$ cells. Cells were acquired on a FACScalibur flow cytometer (BD), gated on CD14-, CD19-, and Via-Probe-negative lymphocytes, and analyzed using CELLQuest software (BD).

Calculation of tetramer-positive $T$ cell frequencies and validation of the method. Frequencies of tetramer-positive $\mathrm{CD} 4^{+} \mathrm{T}$ cells were determined as described previously (23). Briefly, the input number of $\mathrm{CD}^{+} \mathrm{T}$ cells in the enriched sample was calculated by multiplying the number of $\mathrm{CD}^{+}$cells in the pre-enrichment sample ( $10 \%$ of total) by a factor of 9 . The frequency of tetramer-positive cells was thus calculated by dividing the post-enrichment output number of tetramer-positive cells by the calculated input number of $\mathrm{CD} 4^{+}$cells. To determine whether this technique gives a reproducibly linear enrichment recovery, we spiked HIV-negative PBMCs with a CD4+ T cell clone (Ox97 clone 10) specific for the DRB1*0101-restricted p24.17 epitope at frequencies ranging from $0.001 \%$ to $0.5 \%$. These samples were then subjected to HLA class II tetramer staining and magnetic bead enrichment as done for ex vivo analysis.

IFN- $\gamma$ and IL-2 secretion assays. IFN- $\gamma$ and IL-2 production were determined with the IFN- $\gamma$ and IL- 2 Cell Enrichment and Detection Kit (Miltenyi Biotech). We stimulated $2-10 \times 10^{6} \mathrm{PBMCs}$ with $5 \mu \mathrm{g} / \mathrm{ml}$ of peptide for 6 hours. Detection and enrichment of cytokine-positive cells was performed according to the manufacturer's protocol.

Statistical analyses. The paired Student's $t$ test and Mann-Whitney $U$ test were performed using Prism version 3.0a for Macintosh (GraphPad). Differences were considered statistically significant when the $P$ value was less than or equal to 0.05 .

\section{Acknowledgments}

We thank Gillian Harcourt and Andrew K. Sewell for assistance and discussion. This work was supported by a program grant from the Wellcome Trust (to R.E. Phillips and J.N. Weber). T.J. Scriba was funded by the Fogarty Foundation and the South African National Research Foundation. A. Oxenius is supported by the Swiss National Science Foundation, the Vontobel Foundation, and the Roche Research Fund. P. Klenerman is a Wellcome Trust Senior Clinical Research Fellow. C.L. Day is a Royal Society Research-funded fellow. M. Lucas is funded by EU grant QLK2CT 200201329.

Received for publication August 19, 2004, and accepted in revised form November 23, 2004.

Address correspondence to: Rodney E. Phillips, The Peter Medawar Building for Pathogen Research, South Parks Road, Oxford OX1 3SY, United Kingdom. Phone: 44-1865-281230; Fax: 44-1865281890; E-mail: rodney.phillips@ndm.ox.ac.uk.
1. Rosenberg, E.S., et al. 1997. Vigorous HIV-1-specific CD4+ T cell responses associated with control of viremia. Science. 278:1447-1450.

2. McNeil, A.C., et al. 2001. High-level HIV-1 viremia suppresses viral antigen-specific CD4(+) T cell proliferation. Proc. Natl. Acad. Sci. U. S. A. 98:13878-13883.

3. Douek, D.C., et al. 2002. HIV preferentially infects HIV-specific CD4+ T cells. Nature. 417:95-98.

4. Oxenius, A., et al. 2002. Stimulation of HIV-specific cellular immunity by structured treatment interruption fails to enhance viral control in chronic HIV infection. Proc. Natl. Acad. Sci. U. S. A. 99:13747-13752.

5. Davey, R.T., Jr., et al. 1999. HIV-1 and T cell dynam- ics after interruption of highly active antiretroviral therapy (HAART) in patients with a history of sustained viral suppression. Proc. Natl. Acad. Sci.U. S. A. 96:15109-15114.

6. Binley, J.M., et al. 2000. The relationship between T cell proliferative responses and plasma viremia during treatment of human immunodeficiency virus type 1 infection with combination antiretroviral therapy. J. Infect. Dis. 181:1249-1263.

7. Blankson, J.N., Gallant, J.E., and Siliciano, R.F. 2001. Proliferative responses to human immunodeficiency virus type 1 (HIV-1) antigens in HIV-1infected patients with immune reconstitution. J. Infect. Dis. 183:657-661.
8. Markowitz, M., et al. 2002. Discontinuation of antiretroviral therapy commenced early during the course of human immunodeficiency virus type 1 infection, with or without adjunctive vaccination. J. Infect. Dis. 186:634-643.

9. Pontesilli, O., et al. 1999. Lymphoproliferative response to HIV type 1 p24 in long-term survivors of HIV type 1 infection is predictive of persistent AIDS-free infection. AIDS Res. Hum. Retroviruses. 15:973-981.

10. Betts, M.R., et al. 2001. Analysis of total human immunodeficiency virus (HIV)-specific CD4(+) and CD8(+) T-cell responses: relationship to viral load in untreated HIV infection. J. Virol. 75:11983-11991.

11. Palmer, B.E., Boritz, E., Blyveis, N., and Wilson, 
C.C. 2002. Discordance between frequency of human immunodeficiency virus type 1 (HIV-1)specific gamma interferon-producing CD4(+) $\mathrm{T}$ cells and HIV-1-specific lymphoproliferation in HIV-1-infected subjects with active viral replication. J. Virol. 76:5925-5936

12. Pitcher, C.J., et al. 1999. HIV-1-specific CD4+ T cells are detectable in most individuals with active HIV-1 infection, but decline with prolonged viral suppression. Nat. Med. 5:518-525.

13. Rosenberg, E.S., et al. 2000. Immune control of HIV-1 after early treatment of acute infection. Nature. 407:523-526.

14. Iyasere, C., et al. 2003. Diminished proliferation of human immunodeficiency virus-specific CD4+ T cells is associated with diminished interleukin-2 (IL-2) production and is recovered by exogenous IL-2. J. Virol. 77:10900-10909.

15. Younes, S.A., et al. 2003. HIV-1 viremia prevents the establishment of interleukin 2-producing HIV-specific memory CD4+ T cells endowed with proliferative capacity. J. Exp. Med. 198:1909-1922.

16. Palmer, B.E., Boritz, E., and Wilson, C.C. 2004 Effects of sustained HIV-1 plasma viremia on HIV-1 gag-specific CD4+ T cell maturation and function. J. Immunol. 172:3337-3347.

17. Sallusto, F., Lenig, D., Forster, R., Lipp, M., and Lanzavecchia, A. 1999. Two subsets of memory T lymphocytes with distinct homing potentials and effector functions. Nature. 401:708-712.

18. Harari, A., Petitpierre, S., Vallelian, F., and Pantaleo, G. 2004. Skewed representation of functionally distinct populations of virus-specific CD4 T cells in HIV-1-infected subjects with progressive disease: changes after antiretroviral therapy. Blood. 103:966-972.

19. Harari, A., et al. 2002. Analysis of HIV-1- and CMVspecific memory CD4 T-cell responses during primary and chronic infection. Blood. 100:1381-1387.

20. Sallusto, F., et al. 1999. Switch in chemokine receptor expression upon TCR stimulation reveals novel homing potential for recently activated T cells. Eur. J. Immunol. 29:2037-2045.

21. Chao, C.C., Jensen, R., and Dailey, M.O. 1997. Mechanisms of L-selectin regulation by activated T cells. J. Immunol. 159:1686-1694.

22. Day, C.L., et al. 2003. Ex vivo analysis of human memory CD4 $\mathrm{T}$ cells specific for hepatitis $\mathrm{C}$ virus using MHC class II tetramers. J. Clin. Invest. 112:831-842. doi:10.1172/JCI200318509.

23. Lucas, M., et al. 2004. Ex vivo phenotype and frequency of influenza virus-specific CD4 memory $\mathrm{T}$ cells. J. Virol. 78:7284-7287.

24. Lechner, F., et al. 2000. Analysis of successful immune responses in persons infected with hepatitis C virus. J. Exp. Med. 191:1499-1512.

25. Barnes, E., et al. 2004. Ultra-sensitive class I tetramer analysis reveals previously undetectable populations of antiviral CD8+ T cells. Eur. J. Immunol. 34:1570-1577.

26. Meyer, A.L., et al. 2000. Direct enumeration of Borrelia-reactive CD4 T cells ex vivo by using MHC class II tetramers. Proc. Natl. Acad. Sci. U. S. A. 97:11433-11438.

27. Fidler, S., et al. 2002. Virological and immunological effects of short-course antiretroviral therapy in primary HIV infection. AIDS. 16:2049-2054.

28. Mohri, H., et al. 2001. Increased turnover of T lymphocytes in HIV-1 infection and its reduction by antiretroviral therapy. J. Exp. Med. 194:1277-1287.

29. Douek, D.C., et al. 2001. Evidence for increased T cell turnover and decreased thymic output in HIV infection. J. Immunol. 167:6663-6668.

30. Fleury, S., et al. 2000. Long-term kinetics of T cell production in HIV-infected subjects treated with highly active antiretroviral therapy. Proc. Natl. Acad. Sci.U. S. A. 97:5393-5398.

31. Hellerstein, M., et al. 1999. Directly measured kinetics of circulating $\mathrm{T}$ lymphocytes in normal and HIV-1-infected humans. Nat. Med. 5:83-89.

32. Sachsenberg, N., et al. 1998. Turnover of CD4+ and CD8+ T lymphocytes in HIV-1 infection as mea- sured by Ki-67 antigen. J. Exp. Med. 187:1295-1303. 33. Lempicki, R.A., et al. 2000. Impact of HIV-1 infection and highly active antiretroviral therapy on the kinetics of CD4+ and CD8+ T cell turnover in HIV-infected patients. Proc. Natl. Acad. Sci. U. S. A. 97:13778-13783.

34. Novak, E.J., Liu, A.W., Nepom, G.T., and Kwok, W.W. 1999. MHC class II tetramers identify peptide-specific human $\mathrm{CD}^{+} \mathrm{T}$ cells proliferating in response to influenza A antigen. J. Clin. Invest. 104:R63-R67.

35. McMichael, A.J., and Kelleher, A. 1999. The arrival of HLA class II tetramers. J. Clin. Invest. 104:1669-1670.

36. Oxenius, A., et al. 2001. Variable fate of virus-specific CD4(+) T cells during primary HIV-1 infection. Eur. J. Immunol. 31:3782-3788.

37. Deeks, S.G., et al. 2004. Immune activation setpoint during early HIV infection predicts subsequent CD4+ $\mathrm{T}$ cell changes independent of viral load. Blood. 104:942-947.

38. Lichterfeld, M., et al. 2004. Loss of HIV-1-specific CD8+ T cell proliferation after acute HIV-1 infection and restoration by vaccine-induced HIV-1-specific CD4+ T cells. J. Exp. Med. 200:701-712.

39. Sallusto, F., Geginat, J., and Lanzavecchia, A. 2004. Central memory and effector memory $T$ cell subsets: function, generation, and maintenance. Annu. Rev. Immunol. 22:745-763.

40. Oxenius, A., et al. 2000. Early highly active antiretroviral therapy for acute HIV-1 infection preserves immune function of CD8+ and CD4+ T lymphocytes. Proc. Natl. Acad. Sci. U. S. A. 97:3382-3387.

41. Malhotra, U., et al. 2000. Effect of combination antiretroviral therapy on T-cell immunity in acute human immunodeficiency virus type 1 infection. J. Infect. Dis. 181:121-131.

42. Bunce, M., et al. 1995. Phototyping: comprehensive DNA typing for HLA-A, B, C, DRB1, DRB3, DRB4, DRB5 \& DQB1 by PCR with 144 primer mixes utilizing sequence-specific primers (PCR-SSP). Tissue Antigens. 46:355-367. 